SYSTEMATICS

\section{Blind dating}

PLoS ONE 11, e0152394 (2016)

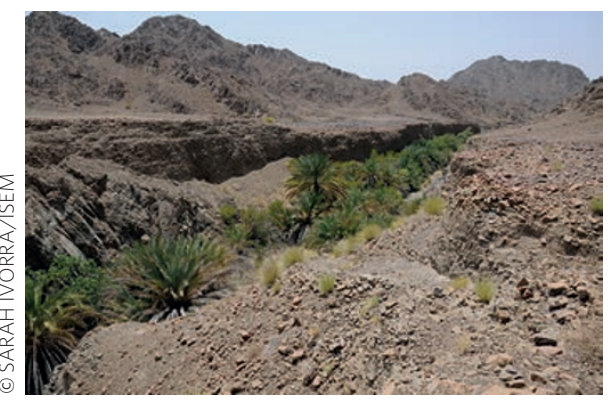

Date palm (Phoenix dactylifera L.) is one of 14 barely distinguishable species in the genus, meaning that determining how, when and where it was domesticated is very difficult. Muriel Gros-Balthazard of the University of Montpellier, France, and colleagues have solved this problem with a morphometric analysis of 1,625 Phoenix seeds from different species.

P. dactylifera is economically important in the Middle East and North Africa, not only for the fruit it produces but also because the trees shelter other crops. Moreover, there are no confirmed wild populations of $P$. dactylifera still living, meaning that archaeobotanists, who rely on modern reference species to characterize archaeological specimens, are not even sure what the wild precursor to modern date palms would look like.

The new method allowed Gros-Balthazard et al. to differentiate between Phoenix species and define their unique characteristics. In particular they found that cultivated date palm seeds are distinctively longer and more elongated in shape than wild species. They used this domestication hallmark to suggest that some uncultivated date palms in Oman, previously thought to be feral, are actually wild. The authors intend to roll out the method for identification of seeds recovered from archaeological sites, such as Miri Qalat and Shahi Tump in Pakistan, to further illuminate the domestication of this key species.

\section{IMMUNITY}

\section{RALF to the rescue}

Nature Microbiol. 1, 16043 (2016)

Biotrophic and hemibiotrophic fungi love an alkaline environment, as it increases their infectious growth. In plants, numerous RALF (rapid alkalinization factor) peptides are involved as endogenous developmental regulators. One of their most striking effects is an increase in apoplastic $\mathrm{pH}$. Antonio Di Pietro of the University of Córdoba, Spain, and colleagues show that, to infect more efficiently, fungi hijack this plant pathway by producing their own RALF peptides.

The root-infecting fungus Fusarium oxysporum causes wilt disease, leading to yield losses in many different crops. Using tomato and Arabidopsis, the authors show that Fusarium and other plant pathogenic fungi contain a functional homologue of plant RALF peptides. Infecting the host plant with a fungal strain lacking the peptide
Germplasm conservation studies tend to focus on managing diversity within centres of origin or ex situ in seed banks. A new study by Joanne Heraty and Norman Ellstrand of the University of California, USA, examines the genetic diversity of maize (Zea mays spp. mays) varieties in home and community gardens planted by immigrant Mexican farmers in southern California.

Heraty and Ellstrand compared the genetic diversity of commercial varieties available in California with that of the varieties actually being grown by the migrants. They saw that the genetic diversity of the 'migrant' maize was similar to that seen in Mesoamerican landraces. This could be the result of multiple introductions, selective breeding and crosspollination among the several introduced varieties. These introductions may represent seeds that the farmers brought with them, or from planting of corn imported from Mexico intended as food.

Given that genetic diversity of maize in Mexico is known to be eroding, the researchers suggest that the small-scale cultivation of the multiple maize varieties Mexican farmers bring to California is an important way of maintaining genetic diversity. They argue that current research into maize biodiversity ignores the importance of human migration and the accompanying food species. Instead, they suggest, we should celebrate the migrant farmers who are conserving maize diversity in new places. reduces external alkalinization and limits the pathogen virulence. Moreover, similarly to the endogenous peptides, the perception of fungal RALF by the plant is mediated by FERONIA, a multifunctional receptor-like kinase.

Fusarium gained the ability to activate a plant developmental pathway by acquiring or mimicking an alkalinization-inducing peptide, and uses it to enhance its own infectious growth by suppressing plant defences. This research could be used for increasing crop resistance to pathogenic fungi. It also highlights the often neglected role of $\mathrm{pH}$ in biological interactions, and further blurs the distinction between pathogen effectors and the more generic non-self signals known as MAMPs.

\section{RNA SILENCING}

\section{Machinery in algae}

Genome Res. 26, 519-529 (2016)

RNA silencing in plants has been studied the most in angiosperms, but Adrian A. Valli and colleagues at the University of Cambridge, UK, have recently investigated the process in the single-cell alga Chlamydomonas reinhardtii using a forward genetic screen.

They used a light-induced cell death reporter system to identify RNA silencing mutants, and characterized three of them with mutations of the DCL3 gene. Genetic complementation of the mutants confirmed that DCL3 has a central role in the biogenesis of both miRNAs and siRNAs. DCL3 differs from plant DCLs but has some limited similarity to the Drosha protein involved in animal miRNA biogenesis.

The DCL3-dependent siRNAs derive from coding genes or transposable elements, as in higher plants. The miRNAs, however, are unlike their higher plant counterparts in that they are generated mostly from introns or untranslated regions of coding RNAs rather than non-coding regions. Another difference is that the C. reinhardtii miRNAs and siRNAs are not negative regulators of mRNA accumulation. The only mRNAs to over-accumulate in the $d c l 3$ mutants were those that had an miRNA precursor structure in the untranslated region.

The unique features of RNA silencing in C. reinhardtii provide insight into the evolution and function of this pathway in eukaryotes. 